\title{
Transmission Expansion Planning - A Multiyear Dynamic Approach Using a Discrete Evolutionary Particle Swarm Optimization Algorithm
}

\author{
M. C. Rocha ${ }^{1, a}$, J. T. Saraiva $a^{2, b}$ \\ ${ }^{1}$ Dept. Eng. Electrotécnica e Computadores da Faculdade Engenharia da Universidade do Porto, Rua \\ Dr. Roberto Frias, 4200 - 465 Porto, Portugal \\ ${ }^{2}$ INESC Porto, Dept. Eng. Electrotécnica e Computadores da Faculdade Engenharia da Universidade \\ do Porto, Rua Dr. Roberto Frias, 4200 - 465 Porto, Portugal
}

\begin{abstract}
The basic objective of Transmission Expansion Planning (TEP) is to schedule a number of transmission projects along an extended planning horizon minimizing the network construction and operational costs while satisfying the requirement of delivering power safely and reliably to load centres along the horizon. This principle is quite simple, but the complexity of the problem and the impact on society transforms TEP on a challenging issue. This paper describes a new approach to solve the dynamic TEP problem, based on an improved discrete integer version of the Evolutionary Particle Swarm Optimization (EPSO) meta-heuristic algorithm. The paper includes sections describing in detail the EPSO enhanced approach, the mathematical formulation of the TEP problem, including the objective function and the constraints, and a section devoted to the application of the developed approach to this problem. Finally, the use of the developed approach is illustrated using a case study based on the IEEE 24 bus 38 branch test system.
\end{abstract}

\section{Introduction}

A dynamic, or multiyear, TEP model aims at determining the timing, the type and the location of the new transmission facilities that should be added to an existing network along a planning horizon in order to ensure an adequate transmission capacity taking into account future generation options and load requirements, in a long term horizon. Even though this principle is quite simple, the complexity of the problem and the impact on society transform TEP on a challenging issue. The next paragraphs enumerate some of the reasons determining the nature of this problem:

- Complexity of the problem - TEP is a complex problem as it has a mixed integer non-linear nature that in fact corresponds to a combinatorial problem. It is also a complex mathematical problem as it involves, typically, a large number of problem variables and parameters, most of them not easily forecasted along the planning horizon;

\footnotetext{
a e-mail : mz.costeiradarocha@gmail.com

be-mail : jsaraiva@fe.up.pt
} 
- Impact on Society - even in small countries, network expansion means important investments. As an example, the Portuguese TSO plans to invest 1.430 million Euros between 2009 and 2014 to modernize and expand its transmission network, integrate new renewable generation centers, increase the interconnection capacity with Spain and enable Portugal to become a net exporter of energy in the medium-term. On the other hand, when computing costs, one has also to consider the costs incurred due to failures of the power system.

On the legal and regulatory side, the European Parliament and the European Council approved the Directive 2003/54/CE, aiming at establishing common rules for the electricity internal market. TEP is clearly identified as a major responsibility of Transmission System Operators, TSO's, as these operators are responsible for ensuring the long-term ability of the system to meet reasonable demands for the transmission of electricity. More recently, the Commission Regulation (EU) No. 838/2010 of 23 September 2010, on laying down guidelines relating to the inter-transmission system operator compensation mechanism states that “(...) the union-wide assessment of electricity transmission infrastructure associated with facilitating cross-border flows of electricity should be carried out by the Agency for the Co-operation of Energy Regulators ${ }^{\mathrm{a}}$ as the body responsible for coordinating the activities of regulatory authorities who must carry out a similar function at a national level."

For many years, the TEP problem was addressed in an integrated way with generation expansion planning, namely before the restructuring of power systems as it is well illustrated in [1]. Then, with the development of the restructuring process, generation and transmission expansion planning problems got decoupled, namely reflecting the existence of different agents and owners of generation assets and of transmission grids. This fundamentally changed the nature of the TEP problem [2] that is now, much more than in the past, influenced by uncertainties affecting, for instance, the evolution of the demand, the requests to connect new generation centers and the impact of the increasing amount of renewables, several of them using volatile resources. Accordingly, the TEP formulations and solution algorithms have to meet new challenges, namely:

- there are typically several entities owning generation assets and other or others operating the transmission networks;

- the capacity of transmission lines determines the degree to which generators in different locations can compete;

- new wind parks developers are already asked to invest themselves on the distribution grid reinforcement;

- there is the substitution effect of transmission by distributed generation or by load via demandside management;

- depending on the size of the distributed generation units, their location and ownership, network can help to reduce market power and thereby lead to a more efficient market;

- there are more and more environmental constraints, such as limits on pollution produced by power plants and on the construction of new lines. These aspects contribute to turn the licensing of new transmission projects increasingly difficult;

- the new organizational structure of the electricity industry leads to new relations between agents, to a decoupling between the flow of electricity and the flow of money, and it requires an unbundling of traditional tariff schemes, namely with the creation of specific tariffs to remunerate network providers for their costs.

On the other hand, for many years the TEP problem was addressed in an approximate way in terms of substituting discrete variables representing the possible investment decisions by continuous ones that are rounded up to integers when finishing solving the resulting mathematical problem. On other cases, the originally multiyear dynamic TEP problem was solved considering a sequence of yearly problems that are addressed in sequence. None of these approaches are adequate in terms of

\footnotetext{
${ }^{\text {a }}$ Established by Regulation (EC) No 713/2009 of the European Parliament and of the Council
} 
translating the true nature of the problem and this was the main motivation for the research that was developed and that is partially reported in this paper.

Having in mind these concerns, this paper is structured as follows. Apart from this introductory section, Section 2 provides an overview on TEP approaches and Section 3 describes the main features of the enhanced EPSO algorithm, namely to include more adequately discrete variables. This enhanced approach is termed as DEPSO, Discrete Evolutionary Particle Swarm Optimization. Section 4 details the mathematical formulation of the TEP problem and Section 5 describes the adopted solution algorithm. Section 6 illustrates the application of this approach to an IEEE test system and finally Section 7 enumerates the main conclusions.

\section{Multiyear Transmission Expansion Planning Model Overview}

As stated above, the objective of the TEP problem is to determine the timing, the type and the location of new transmission elements to add to an existing transmission network along a planning horizon. The selection of these new assets should be driven by a number of criteria and should also enable the network to integrate future generation additions as well as the forecasted demand evolution under a specified reliability level. When mentioning future generation it is important to consider not only traditional centralized generation centers but also generation using volatile resources (as, for instance, wind parks and solar PV stations) directly connected to transmission networks or to distribution grids. In both cases, either in a direct way or indirectly in terms of the liquid demand seen by transmission networks, the impact on transmission grids is very relevant. This means that transmission networks should be prepared to accommodate connection requests and to operate more decentralized generation systems in a safe and reliable way. The literature on TEP is vast and $[2,3]$ enumerate and classify publications on this problem. In the next paragraphs some of the key features of TEP will be briefly addressed.

It is important to distinguish between static and dynamic formulations. Static formulations as the ones in $[4,5]$ are approaches in which the time steps in the horizon are treated in a separate and sequential way. This means that one solves consecutively planning problems for each step in the horizon and the additions identified in a particular sub period are assumed as available is posterior periods. The final plan just corresponds to the addition of a number of partial plans. This solution strategy loses the global view that should exist over the problem, in the sense that the solution of a true multiyear problem will not, in general, be the same as the collection of the partial individual year plans. Dynamic or multiyear approaches explicitly include information regarding the entire planning horizon represented by a number of periods or stages. In these models, the expansion plan is outlined along the entire horizon in a multistage and coordinated way. In this sense, [6, 7] describe multiyear approaches of the TEP problem. Given the complexity of the fully integrated problem, several authors proposed simplifications based for instance in series of static sub problems leading to formulations often termed as pseudo-dynamic procedures, as the ones in $[1,8]$.

TEP models use several optimization techniques including classical optimization methods, dynamic and quadratic programming, mix-integer and decomposition techniques and meta heuristics. Regarding meta heuristics, [4, 9] use Genetic and Evolutionary algorithms, Simulated Annealing is used in $[7,10]$, Tabu Search is adopted in [4, 11], Expert Systems are used in [5, 12] and reference [8] details the use of Greedy Randomized Procedures. Finnaly, the operation and the expansion planning of power systems is more than ever before contaminated by uncertainties and [13 - 15] detail some approaches to the TEP problem using probabilistic and fuzzy set models. 


\section{Discrete Evolutionary Particle Swarm Optimization Algorithm}

\subsection{Heuristic tools}

Heuristic methods go step-by-step generating, evaluating, and selecting solutions, with or without interacting with the planner [3]. Taking advantage from planers experience inputs, the computational performance of heuristic methods is usually better than that of the mathematical methods. In some cases, local searches are performed, following rules defined by the planer. The solutions are classified according to these rules, which can be either logical or empirical, and consider different information like technical, financial and service data. The search process is stopped when no further improvement on the best so far found solution was obtained for a specified number of iterations. Heuristic tools include, among others, evolutionary algorithms and particle swarm optimization.

Evolutionary algorithms are usually organized in the following steps:

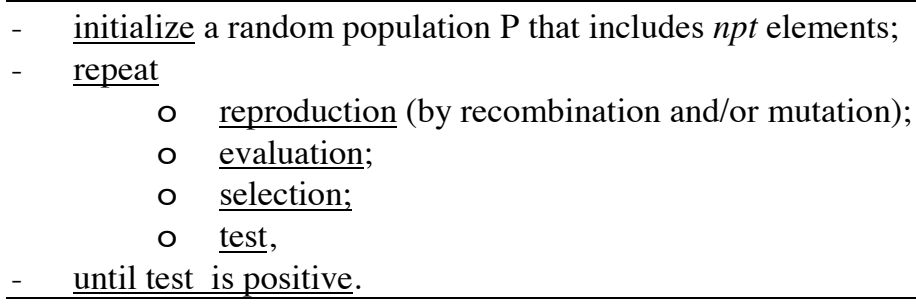

The termination criteria can be based on the value of the fitness function, on the number of iterations or other to be specified.

Evolutionary computation offers several advantages when facing difficult optimization problems [16]: conceptual simplicity, broad applicability, outperform classic methods on real problems, potential to use knowledge and hybridize with other methods, parallelism, robust to dynamic changes and capability for self-optimization. The classical particle swarm optimization (PSO) was proposed in 1995 [17], based on the parallel exploration of the search space by a set of "particles", the solutions or alternatives that are successively transformed along the process.

\subsection{Evolutionary Particle Swarm Optimization, EPSO}

In 2002 it was introduced the Evolutionary Particle Swarm Optimization (EPSO) algorithm [18] joining the best features of both particle swarm methodologies and evolutionary algorithms. The structure of the EPSO algorithm is summarized below.

Procedure EPSO

Initialize a random population $\mathrm{P}$ of $n p t$ particles

Repeat

$\frac{\text { Replication }}{\text { Mutation }}$
$\frac{\text { Reproduction }}{\text { Selection }}$
$\underline{\text { Test }}$

Until test is positive

End EPSO

EPSO focuses in regions of the search space where better contributions for the solution can be found, instead of conducting a blind sampling of the space. More detailed information about EPSO 
can be found in [18] and [19]. In brief, in the EPSO algorithm each particle in the swarm evolves from iteration $k$ to iteration $k+1$ according to expressions (1) and (2). The movement rule of the EPSO algorithm is illustrated in Figure 1.

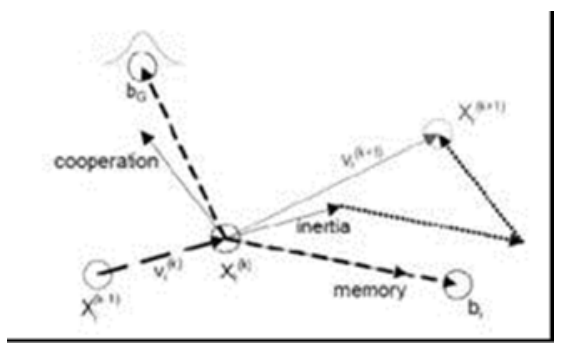

Fig. 1. Illustration of the movement of particle $i$ from iteration $k$ to iteration $k+1$.

$$
\begin{gathered}
X_{i}^{k+1}=X_{i}^{k}+V_{i}^{k+1} \\
V_{i}^{k+1}=W_{i 1} * \cdot V_{i}^{k}+W_{i 2}^{*}\left(b_{i}-X_{i}^{k}\right)+W_{i 3} *\left(b_{G} *-X_{i}^{k}\right) \cdot P
\end{gathered}
$$

In these expressions:

$X_{i}^{k}$
$X_{i}{ }^{k+1}$
$b_{i}$
$b_{G}{ }^{*}$
$V_{i}^{k}=X_{i}^{k}-X_{i}^{k-1}$
$W_{i 1}{ }^{*}$
$W_{i 2}^{*}$
$W_{i 3^{*}}$
$W_{i 4^{*}}$
$P$

- location of the particle $i$ in generation $k$;

- location of the particle $i$ in generation $k+1$;

- best point found by particle $i$ in its past life up to the current generation;

- best global point found by the swarm of particles in their past life up to the current generation;

- velocity of particle $i$ at generation $k$;

- weight conditioning the inertia term;

- weight conditioning the memory term;

- weight conditioning the cooperation term;

- weight conditioning the best global particle;

- communication factor.

According to (1), the position of particle $i$ in iteration $k+1$ is determined by its position in iteration $k$ and by the so called velocity vector, $V_{i}^{k+1}$. This vector typically integrates three terms as follows:

- the inertia term, that translates the influence in the move from iteration $k$ to $k+1$ of particle $i$ obtained in the previous iteration;

- the memory term, that uses information about the best of the ancestors of this particle, $b_{i}$;

- the cooperation term, that tends to attract the particle to the best point ever found along the iterative process, $b_{G}{ }^{*}$. This term is multiplied by a communication factor $P$ in the range $[0,1]$ that is used to enable transmitting information to particle $i$ only from some dimensions of the best global particle.

These terms are all multiplied by the corresponding inertia, memory and cooperation weights. These weights are set in the beginning of the algorithm and are then mutated in each iteration, for instance using (3). In this expression, $\tau$ is a learning parameter set in the beginning of the iterative process. Similarly, the best global particle ever found along the iterative process also undergoes mutation, for instance using (4).

$$
\begin{aligned}
& W_{i}^{*}=W_{i} \cdot[\log N(0,1)]^{\tau} \\
& b_{G}^{*}=b_{G}+W_{i 4}^{*} \cdot N(0,1)
\end{aligned}
$$




\subsection{Discrete Evolutionary Particle Swarm Optimization, DEPSO}

The approach introduced in this paper is based on a discrete modeling of EPSO that we termed as DEPSO, Discrete Evolutionary Particle Swarm Optimization. The possible solutions of the problem under analysis are represented by vectors of integers and the off-springs are generated by recombination of the particles, following the classical EPSO rules as indicated by (1) and (2). However, the developed DEPSO differs from the EPSO algorithm in a number of items as follows:

- the weights used in the inertia, memory and cooperation terms are mutated using expressions different from (3). In our case, we used expressions including the logistic function as a way to turn the search more chaotic given that several researchers concluded that this behavior favors the improvement of the characteristics of the swarm. In particular, the inertia, the memory and the cooperation weights are mutated using (5);

$$
w_{i}^{k+1 *}=\left(0.5+\operatorname{rand}()-\frac{1}{1+\exp \left(-w_{i}^{k^{*}}\right)}\right)
$$

- secondly, once all the weights are mutated, we use a similar recombination rule as the EPSO but each position in the velocity vector of particle $i$ determining its move from iteration $k$ to iteration $k+1$ is rounded to the nearest integer leading to (6). When doing this rounding procedure it is possible to obtain integer values outside the admissible set of integers. If that happens, it is adopted a repair strategy and outliers are returned back to the search space substituting the integer outside the admissible range by the nearest admissible integer.

$$
V_{i}^{k+1}=\operatorname{round}\left(w_{i, 1}^{k+1^{*}} \cdot V_{i}^{k}+w_{i, 2}^{k+1^{*}} \cdot\left(b_{i}-X_{i}^{k}\right)+w_{i, 3}^{k+1^{*}} \cdot\left(b_{G}^{*}-X_{i}^{k}\right) \cdot P\right)
$$

- thirdly, since we are working with integers it is possible to obtain a zero vector for $V_{i}^{k+1}$. If that happens, the particle would not move from iteration $k$ to iteration $k+1$. In order to introduce more diversity in the search, it was used in these cases a Lamarkian inspired movement acting directly on some dimensions of the velocity vector. This means that instead of changing some genotype elements of the particle inducing changes on it, we act at the fenotype level sampling some dimensions of the particle to be mutated;

- finally, in each iteration of the algorithm we work with two populations. The first population is initially randomly built and then, in each iteration, this population is cloned and all the particles in both populations are subjected to the mutation and the movement rules. Then, all the particles are evaluated using the fitness function defined for the problem under analysis. Using the fitness values, we finally perform a selection step comparing particle $i$ of population 1 with particle $i$ of population 2 and passing to the position $i$ of the population in the next iteration the one having a smaller fitness in case of minimization problems. This new population is cloned so that we start a new iteration using again two populations.

\section{Mathematical Formulation of the Multiyear TEP Problem}

\subsection{Project list, possible solutions and search space}

The search space under analysis is discrete and it typically includes a large number of possible alternative projects given by $n p^{n p r}$, where $n p$ is the number of periods and $n p r$ is the number of possible individual projects. Each new project, a new line or a new transformer, is defined by the following information: origin node, destination node, technical data and investment $(€)$. A solution $X_{i}$ of the TEP problem corresponds to a plan that includes a number of projects selected among this list as well as their location in the time frame. On the other hand, a particle is modelled as a vector of $n p r$ positions, each one related to a project in the list. Each $n p r$ position has information about the 


\section{$2^{\text {nd }}$ European Energy Conference}

location in the time frame of that particular project, that is, it has an integer ranging from 1 to $\mathrm{np}$. In fact, apart from integers from 1 to $\mathrm{np}$, one also allows two other integer values:

- $\quad 0$ meaning that the project was not selected to integrate the plan modelled by that particle;

- $\quad n p+1$ meaning that the project was postponed.

Allowing these two extra integers, and in particular $n p+1$, means that the set of admissible integers are balanced since we are allowing both an integer below the lowest possible value, that is below 1, and one integer larger than the last possible period of the planning horizon. If the integer $n p+1$ was not allowed, when obtaining a value larger than $n p$ we would have to return it back to $n p$ and this could distort the final plan in terms of including an excessive number of projects in the last period of the horizon. Allowing $n p+1$ just balances this situation turning it similar to the cases in which the recombination rules of the DEPSO lead to 0 values, for instance.

\subsection{TEP modelling}

The general formulation of the TEP problem is given by (7-10). The list of possible projects mentioned in 4.1 may include not only new lines to establish in new corridors, but also lines to install in existing corridors or projects associated with the upgrade of the capacity of existing lines, for example increasing the voltage level. Each of these projects $j$ is characterized by its investment cost $I C_{j}$. Then, if a particular project $j$ is selected for a particular year $p$, there is a binary variable $K_{i j}^{p}$ that is set to 1 , and its investment cost is referred to the year 0 , using an interest rate ir appropriate to the risk of this type of investment. On the other hand, for a given particle $i$ in iteration $k$, there are a number of assets available in period $p$. Using these elements, we estimate the operation costs $O C_{p i}$ and return them back to the initial period using the interest rate $i r$. The objective function (7) is subjected to a number of constraints related with the physical characteristics of the generation and transmission assets in the system, to financial limitations of the transmission company and to quality of service and reliability constraints.

$$
\min \operatorname{Cost} X_{i}^{k}=\sum_{p=0}^{n p+1}\left[\sum_{j=1}^{n p r} I C_{j} \cdot K_{i j}^{p}+O C_{p i}\right] /(1+i r)^{p}
$$

Subject to: $\quad$ Physical constraints (generation; power flow limitations);

Financial constraints (global and period constraints);

Quality of service constraints (reliability)

Operation Costs in (7) are evaluated solving a linearized DC_OPF problem as (11-15) for each period. In each period the network integrates the installations commissioned previously and the forecasted demand. In this formulation $c_{k}, P g_{k}$ and $P l_{k}$ represent the variable generation cost, the generation level and the load connected to node $k, G$ is a penalty assigned to Power Not Supplied, PNS, $a_{b k}$ is the sensitivity coefficient of the active flow in branch $b$ regarding the injected power in node $k, P g_{k}^{\min }$ and $P g_{k}^{\max }$ are the minimum and the maximum output of the generator connected to node $k$ and finally $P_{b}^{\min }$ and $P_{b}^{\max }$ are the minimum and the maximum active power flow in branch $b$.

$$
\begin{aligned}
\min f & =\sum c_{k} \cdot P g_{k}+G \cdot \sum P N S_{k} \\
\text { subj } \quad & \sum P g_{k}+\sum P N S_{k}=\sum P l_{k} \\
& P g_{k}^{\text {min }} \leq P g_{k} \leq P g_{k}^{\max } \\
& P N S_{k} \leq P l_{k} \\
& P_{b}^{\text {min }} \leq \sum a_{b k} \cdot\left(P g_{k}+P N S_{k}-P l_{k}\right) \leq P_{b}^{\text {max }}
\end{aligned}
$$


This DC-OPF model was enhanced to include an estimate of transmission losses according to the following scheme.

$\underline{\text { Algorithm }}$

i) Run an initial dispatch using (11) to (15);

ii) Compute voltage phases using the DC model;

iii) Estimate active losses in branch $m-n$ using (16). In this expression, $g_{m n}$ is the conductance of branch $m-n$ and $\theta_{m n}$ is the phase difference across this branch;

$$
\operatorname{Loss}_{\mathrm{mn}} \approx 2 \cdot \mathrm{g}_{\mathrm{mn}} \cdot\left(1-\cos \theta_{\mathrm{mn}}\right)
$$

iv) Add half of the losses in every branch $m-n$ to the original loads in nodes $m$ and $n$. Run a new dispatch using (11) to (15) and compute voltage phases;

v) End if the difference of voltage phases in all nodes is smaller than a specified threshold. If not, return to iii).

The convergence of this iterative process is usually reached in less than 5 iterations and at the end we get the generation cost, the level of losses and eventually a non-zero value for Power Not Supplied.

\subsection{Constraints}

The formulation (7-10) includes a number of constraints as follows: physical limits of the network, physical limits of the generators, financial limitations of the transmission provider, number of projects that can be developed simultaneously, value of a reliability index to ensure the quality of the expansion plan. Several of these constraints are inherently considered in the DC-OPF formulation (11-15). This includes the limits of the branches and of the generators. On the other hand, if a particular plan displays in a particular year of the horizon a non-zero value of PNS then this is penalized in (11) and so the global operation cost of this particle also becomes penalized.

To turn the problem more realistic we also admitted that the planner can specify limits associated with the financial resources and with the number of projects that can be implemented simultaneously. Regarding the financial limitations we can specify a maximum value for the yearly investment cost and, if this value is exceeded for a particular plan, then the corresponding cost is penalized.

Regarding the reliability evaluation, the developed approach penalizes plans in which the PNS is non-zero for configurations of the network associated to $\mathrm{N}-1$ contingencies and for a selected number of N-2 contingencies. This follows the indications in the Grid Codes of several countries that explicitly indicate that the system should be able to supply the demand for all $\mathrm{N}-1$ contingencies and for a number of $\mathrm{N}-2$ contingencies selected according specific criteria. This evaluation can be modified, extending the number of configurations to analyse or, in the limit, to run a Monte Carlo simulation for every particle. This strategy would lead to a dramatic increase of the computation time. These penalty terms are introduced in the objective function of the problem using large values for the penalty coefficients.

\subsection{Evaluation function}

As a result of all the penalty terms mentioned above, the function to be used to evaluate the possible plans is given by (17).

$$
\operatorname{Cost} X_{i}^{k}=\sum_{p=0}^{n p+1}\left[\sum_{j=1}^{n p r} I C_{j} \cdot K_{i j}^{p}+O C_{p i}\right] /(1+i r)^{p}+\sum_{y=1}^{5} \alpha_{y}
$$




\section{$2^{\text {nd }}$ European Energy Conference}

This expression is used to characterize each particle in the populations under analysis and to determine the selection of particles that survive to the next generation. This expression includes operation and investment costs along the horizon referred to the initial year using a discount rate $i r$, assuming that the planning horizon is multiyear. Investment costs, $I C_{j}$ result from the sum of the updated values for each project $j$ included in the solution under analysis, considering the interest rate defined in the beginning of the process. This expression also includes the penalty terms mentioned above, as now summarized:

- $\quad$ a penalty $\alpha_{1}$ is applied if the level of losses estimated from the DC_OPF problem detailed above exceeds a reference value;

- in a similar way, the solution of the DC-OPF algorithm (11) to (15) also outputs the value of PNS. If the PNS(N) is not zero, then the penalty term $\alpha_{2}$ is introduced in the fitness function (17);

- financial constraints - two financial constraints were considered in the model. The first one corresponds to the maximum number of projects that can be implemented per period. This limitation arises due to financial or operational reasons and if it is violated we consider a penalty term $\alpha_{3}$ in the fitness function (17). The second one corresponds to the maximum investment value over the entire horizon and it models a global financial constraint. If this limit is violated a penalty term $\alpha_{4}$ is included in the fitness function (17);

- quality of service and reliability constraints - the developed approach penalizes plans in which the PNS is non-zero for configurations of the network associated to $\mathrm{N}-1$ contingencies and, if necessary, for a selected number of $\mathrm{N}-2$ contingencies. These penalties are considered using the term $\alpha_{5}$ in (17).

\section{Application of the DEPSO to the TEP Problem}

After defining the data characterizing the network under analysis in the departing year and building the list of possible transmission investment projects, one proceeds to the DEPSO settings and eventually to select a particle to be used for seeding purposes, which may accelerate the convergence. The first population is generated with so many randomly defined particles as those associated with the size of the population. Then the multiyear TEP algorithm evolves as follows.

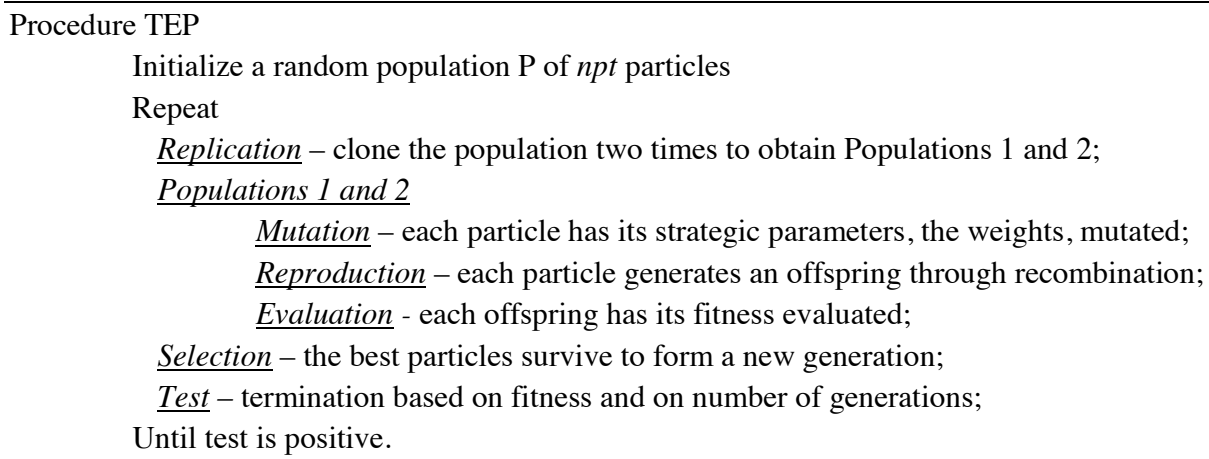

The TEP algorithm ends when the value of the fitness function of the best global particle didn't improve at least by a specified percentage for a number of iterations set in the beginning. On the other hand, it is also typical to set a minimum number of iterations that should be completed before stopping the algorithm as well as a maximum number of iterations after which the algorithm stops. If 
stopping because the maximum number of iterations was reached, the best global particle identified so far will eventually not yet display the most adequate characteristics suggesting that:

- more iterations should be done;

- or the limits imposed to some constraints are too rigid;

- or some more projects should be added to the project list to solve some particular bottlenecks in the transmission network so that building the expansion plan becomes more flexible.

\section{Case Studies}

\subsection{Initial tests}

The first tests using the developed TEP formulation and the DEPSO algorithm were performed on the Garver network, described in the seminal paper [20]. This is a 6 bus network that is very much used by researchers on TEP issues as a test system and for which there are numerous publications and results provided by different solving approaches namely for comparison purposes. In our case, the DEPSO algorithm displayed very interesting characteristics both regarding the reliability of its convergence in terms of identifying the best possible plan in different runs and also in terms of its speed. For instance, we obtained identification rates of the best expansion plan larger than $90 \%$ using populations with at least 30 particles and running 15 iterations in the case of single period analysis and 45 iterations for a four period expansion horizon. The results of these tests are fully reported in [21].

\subsection{Application to the IEEE RTS 24 bus test system}

\subsubsection{Data}

Taking advantage of the extensive tests briefly mentioned in Section 6.1 and of the settings selected for several parameters of the DEPSO algorithm, in a second step we used the IEEE RTS 24 bus test system as a Case Study. Figure 2 displays the single line diagram of this system originally described in [22] and including 38 branches and 32 generators. Regarding this system we used the branch data (namely resistance, reactance and maximum active power flow) specified in [22]. In order to obtain a more stressed transmission system, we increased the total demand to $8.550 \mathrm{MW}$ and the total installed generation capacity is $10.215 \mathrm{MW}$, which correspond to 3 times more than the original values in [22].

Apart from this data, we specified a list of possible projects as shown in Table 1. This list contains 28 possible new transmission aditions, some of them corresponding to new transformers and some other to new overhead lines. As a result, the search space has a dimension of $3^{28}=2,28 x$ $10^{13}$ positions for the single period analysis, and $6^{28}=6,141 \times 10^{21}$ positions, for the four period study. The update of costs to period 0 was made at a $10 \%$ rate per period. The solutions which did not respect either the physical constraints, or PNS(N)>0, or PNS(N-1) $>0 \%$ of the load, were penalized as indicated in Section 4.

Finally, when running the four period study we admitted that the demand would evolve at a rate of $5 \%$ per year and we also considered that there would be two new generation stations to be connected to the network in order to follow the demand increase. These two generators are connected to buses 4 and 19 and have an installed capacity of $300 \mathrm{MW}$ (similar to the generators in bus 7) and $591 \mathrm{MW}$ (similar to the generators in bus 13), respectively. 


\section{$2^{\text {nd }}$ European Energy Conference}

\subsubsection{Single period analysis}

We ran the DEPSO algorithm under different conditions, namely considering different population sizes of 10,30 and 100 particles. From these tests we concluded that a population of just 10 particles was too short in terms of its diversity in order to obtain an adequate frequency of identification of the best expansion plan after running several tests, each one with 1000 iterations. For a population size of 30 particles, we obtained a frequency of identification of the best plan of $95 \%$ after running in average 900 iterations and if using a population with 100 particles then only 590 iterations were in average required to obtain the frequency of $95 \%$.

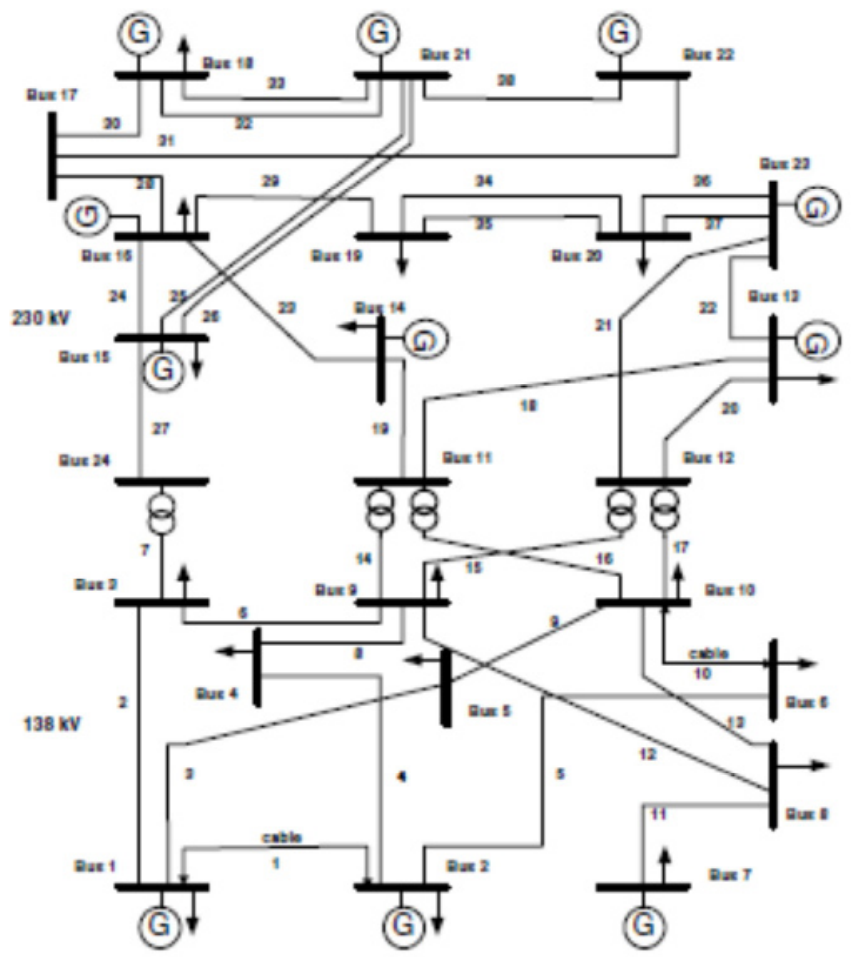

Fig. 2. Single line diagram of the IEEE RTS 24 bus test system.

The best identified plan has an investment cost of 1280,0 M€ and it includes two new branches connecting nodes 1 and 5, one new branch from node 6 to 10, two new branches connecting nodes 7 to 8 and one new branch from node 16 to 17 .

It is still important to mention that for a population of 30 particles, the DEPSO algorithm analyzed 30 particles $\times 2$ populations $\times 900$ iterations $=54000$ possible expansion plans. This compares very favorably with the size of the search space given that in this case this size is given by $3^{28} \approx 2,288.10^{13}$. Finally, these results also compare favorably with the ones reported in [23, 24] namely considering the investment cost reported in these papers for similar running conditions.

\subsubsection{Multi period analysis}

Finally, we applied the DEPSO to the same IEEE 24 bus system but now considering a 4 year analysis. In this case the search space has a dimension of $6^{28} \approx 6,141.10^{21}$ possible combinations of the 28 additions in the project list. We ran the algorithm using population sizes of 30, 100 and 150 particles and it was possible to conclude that the 150 particle test yields the expansion plan having 
the most reduced fitness value requiring an investment cost of 2427,72 $\mathrm{M} €$ and 2700 iterations to converge. The 100 particle test identified the second best expansion plan with an investment cost of 2527,44 M€ after running 1210 iterations while the 30 particle population retrieves the most costly plan with an investment cost of 2599,16 M€ after 320 iterations. Table 2 detail the projects included in these three expansion plans together with the plan associated with the single year analysis detailed in Section 6.2.2. For the branches considered in this table, we indicate the commissioning year in the respective plan. The projects in Table 1 not showed in Table 2 were not selected to integrate any of these four plans.

Table 1. List of projects for the IEEE RTS 24 bus test system.

\begin{tabular}{|c|c|c|c|c|c|c|}
\hline $\begin{array}{c}\text { Branch } \\
\text { no }\end{array}$ & $\begin{array}{c}\text { From } \\
\text { bus }\end{array}$ & $\begin{array}{l}\text { To } \\
\text { bus }\end{array}$ & $\begin{array}{l}\text { Resist. } \\
(\mathrm{pu})\end{array}$ & $\begin{array}{c}\text { React. } \\
(\mathrm{pu})\end{array}$ & $\begin{array}{c}\text { Capacity } \\
\text { (MW) }\end{array}$ & $\begin{array}{l}\text { Cost } \\
\left(10^{6} €\right)\end{array}$ \\
\hline 1 & 3 & 24 & 0,0000 & 0,4195 & 400 & 500 \\
\hline 2 & 9 & 11 & 0,0000 & 0,4195 & 400 & 500 \\
\hline 3 & 10 & 11 & 0,0000 & 0,4195 & 400 & 500 \\
\hline 4 & 10 & 12 & 0,0000 & 0,4195 & 400 & 500 \\
\hline 5 & 1 & 5 & 0,1090 & 0,4225 & 175 & 220 \\
\hline 6 & 1 & 5 & 0,1090 & 0,4225 & 175 & 220 \\
\hline 7 & 2 & 4 & 0,1640 & 0,6335 & 175 & 330 \\
\hline 8 & 2 & 4 & 0,1640 & 0,6335 & 175 & 330 \\
\hline 9 & 2 & 6 & 0,2485 & 0,9600 & 175 & 500 \\
\hline 10 & 2 & 6 & 0,2485 & 0,9600 & 175 & 500 \\
\hline 11 & 6 & 10 & 0,0695 & 0,3025 & 175 & 160 \\
\hline 12 & 7 & 8 & 0,0795 & 0,0032 & 175 & 160 \\
\hline 13 & 7 & 8 & 0,0795 & 0,0032 & 175 & 160 \\
\hline 14 & 8 & 10 & 0,2135 & 0,8255 & 175 & 430 \\
\hline 15 & 11 & 13 & 0,0305 & 0,2389 & 500 & 660 \\
\hline 16 & 12 & 13 & 0,0305 & 0,2380 & 500 & 660 \\
\hline 17 & 14 & 16 & 0,0250 & 0,1945 & 500 & 540 \\
\hline 18 & 15 & 21 & 0,0315 & 0,2450 & 500 & 680 \\
\hline 19 & 15 & 24 & 0,0335 & 0,2595 & 500 & 720 \\
\hline 20 & 16 & 17 & 0,0165 & 0,1295 & 500 & 360 \\
\hline 21 & 16 & 17 & 0,0165 & 0,1295 & 500 & 360 \\
\hline 22 & 16 & 19 & 0,0150 & 0,1150 & 500 & 320 \\
\hline 23 & 17 & 18 & 0,0090 & 0,0720 & 500 & 200 \\
\hline 24 & 20 & 23 & 0,0140 & 0,1080 & 500 & 300 \\
\hline 25 & 11 & 13 & 0,0305 & 0,2380 & 500 & 660 \\
\hline 26 & 12 & 13 & 0,0305 & 0,2380 & 500 & 660 \\
\hline 27 & 11 & 14 & 0,0305 & 0,2380 & 500 & 580 \\
\hline 28 & 14 & 16 & 0,0250 & 0,1945 & 500 & 540 \\
\hline
\end{tabular}


Table 2. Best identified expansion plans obtained in different conditions.

\begin{tabular}{|c|c|c|c|c|c|c|c|c|c|c|c|c|}
\hline \multirow{2}{*}{ Test } & \multirow{2}{*}{$\begin{array}{c}\text { Inv. Cost } \\
(\mathrm{M} €)\end{array}$} & \multicolumn{11}{|c|}{ Expansion projects included in the best identified plans } \\
\hline & & $3-24$ & $10-12$ & $1-5$ & $1-5$ & $2-6$ & $6-10$ & $7-8$ & $7-8$ & $11-13$ & $11-13$ & $16-17$ \\
\hline $\begin{array}{l}4 \text { periods } \\
30 \text { part. }\end{array}$ & 2599,16 & -- & 1 & 1 & -- & 2 & 1 & 1 & 2 & 1 & 3 & -- \\
\hline $\begin{array}{l}4 \text { periods } \\
100 \text { part. }\end{array}$ & 2527,44 & -- & 1 & 1 & -- & 4 & 1 & 1 & 2 & 1 & 3 & -- \\
\hline $\begin{array}{l}4 \text { periods } \\
150 \text { part. }\end{array}$ & 2427,72 & 4 & 1 & 1 & -- & -- & 1 & 1 & 2 & 1 & -- & 4 \\
\hline $\begin{array}{l}1 \text { period } \\
100 \text { part. }\end{array}$ & 1280,00 & -- & -- & 1 & 1 & -- & 1 & 1 & 1 & -- & -- & 1 \\
\hline
\end{tabular}

These results reveal that a multi period transmission planning exercise is not just a collection of yearly exercises in which the additions commissioned in one period become available in the next ones. In fact, the plan identified for the single period analysis doesn't include any branch between nodes 10 and 12 and nodes 11 and 13 while such branches are included in all the plans identified with the 4 period tests. On the other hand, the single year analysis suggests two branches between nodes 1 and 5 while all multiyear plans include just one. Building a branch connecting nodes 16 and 17 is included in the single year analysis and in just one of the 4 year plans, and in this case in period 4. Finally, building the second branch from node 7 to 8 is delayed to period 2 when going from the single year to the 4 year analysis. This turns it clear that performing a multiyear study provides the planner more insight on the problem and turns the solution more flexible given that the algorithm looks at all the years in the horizon at the same time, thus providing a more holistic view on how the bottlenecks of the transmission grid along the horizon can be solved.

\section{Conclusions}

This paper addresses the transmission expansion planning problem, considering that the planners and the solution approaches should maintain an holistic view over the problem. This means that in our view TEP problems should be addressed on a multiyear basis so that the most adequate solutions can be identified profiting from the meshed nature of transmission networks, in the sense that a new addition designed to solve a particular problem in the grid can then be useful to alliviate constraints elsewhere. This multiyear approach ultimately means that less costly solutions can be obtained thus contributing to reduce the regulated costs that, at the end, are passed to electricity consumers via access tariffs.

Finally, this paper also describes the experience gained in using an enhancement on the EPSO algorithm in order to turn it more suited to address integer problems. The results that were obtained so far are very promising both in terms of the quality of the solution and regarding the number of iterations required to get convergence. On going research on this topic is related with the integration of uncertainties in the developed model so that we finally build a more realistic planning tool regarding an area that is crucial to maintain the security and the reliability of power systems.

\section{References}

1. M. V. Pereira, L. M. V. G. Pinto, S. H. Cunha, G. C. Oliveira, "A Decomposition Approach to automated Generation/transmission Expansion Planning”, IEEE Transactions on PAS, PAS-104 (1985), pp. $3074-3083$.

2. C. W. Lee, S. K. K. Ng, J. Zhong, F. F. Wu, "Transmission Expansion Planning From Past to Future", in Proceedings of the IEEE 2006 Power Systems Conference and Exposition, Atlanta, pp. $257-265$.

3. G. Latorre, R. D. Cruz, J. M. Areiza, A. Villegas, "Classification of publications and models on transmission expansion planning“, IEEE Trans. on Power Systems, 18 (2003), pp 938-946. 
4. A. Sadegheih, P. Drake, "System Network Planning Expansion Using Mathematical Programming, Genetic Algorithms and Tabu Search", Energy Conversion and Management, 49 (2008), pp. $1557-1566$.

5. R. K. Gajbhiye, "An Expert System Approach for Multi-Year Short Term Transmission Expansion Planning: An Indian Experience”, IEEE Transactions on Power Systems, 23 (2008), pp. $226-237$.

6. A. H. Escobar, R. A. Gallego, R. L. Romero, "Multi stage and Coordinated Planning of the Expansion of Transmission Systems", IEEE Trans. on Power Systems, 19 (2004), pp. 735 - 744.

7. A. S. D. Braga, J. T. Saraiva, "A Multiyear Dynamic Approach for Transmission Expansion Planning and Long-Term Marginal Costs Computation", IEEE Transactions on Power Systems, 20 (2005), pp. 1631 - 1639.

8. S. Binato, G. C. Oliveira, J. L. Araújo, “A Greedy Randomized Adaptive Search Procedure for Transmission Expansion Planning”, IEEE Trans. on Power Systems, 16 (2001), pp. 247 - 253.

9. R. Pringles, V. Miranda, F. Garcês, "Optimal Expansion of the Transmission System Using EPSO", (in Spanish), in Proceedings of the VII Latin American Congress on Electricity Generation \& Transmission, Chile, 2007.

10. R. Romero, R. A. Gallego, A. Monticelli, "Transmission System Expansion Planning by Simulated Annealing", IEEE Transactions on Power Systems, 11 (1996), pp. 364 - 369.

11. R. A. Gallego, R. Romero, A. Escobar, "Tabu Search Algorithm for Network Synthesis”, IEEE Transactions on Power Systems, 15 (2000), pp. 490 - 495.

12. R. Teive, E. L. Silva, L. Fonseka, “A Cooperative Expert System for Transmission Expansion Planning of Electrical Power Systems”, IEEE Transactions on Power Systems, 13 (1998), pp. $636-642$.

13. H. Cheng, H. Zhu, M. Crow, G. Sheblé, "Flexible Method for Power Network Planning Using the Unascertained Number", Electric Power Systems Research, 68 (2004), pp. 41 - 46.

14. A. S. D. Braga, J. T. Saraiva, "Long Term Marginal Prices - Solving the Revenue Reconciliation Problem of Transmission Providers", in Proceedings of the $15^{\text {th }}$ Power Systems Computation Conference, PSCC 2005, Liege 2005.

15. J. Alvarez, K. Ponnambalam, V. H. Quintana, "Transmission Expansion Under Risk Using Stochastic Programming", in Proceedings of the $9^{\text {th }}$ PMAPS Conference, Stockholm, 2006.

16. D. B. Fogel, "Introduction to Evolutionary Computation", in Modern Heuristic Optimization Techniques: theory and applications to power systems, John Wiley \& Sons, Inc., Hoboken, New Jersey, Chapter 1, pp. 3-20, 2008.

17. J. Kennedy, R. C. Eberhart, "Particle Swarm Optimization", in Proceedings of the 1995 IEEE International Conference on Neural Networks, Perth, Australia, 4 (1995), pp. 1942-1948.

18. V. Miranda, N. Fonseca, "EPSO - Evolutionary Particle Swarm Optimization, a New Algorithm with Applications in Power Systems", in Proceedings of the 2002 IEEE Transmission and Distribution Asia-Pacific Conference, Yokohama, 2 (2002), pp 745-750.

19. V. Miranda, H. Keko, A. Jaramillo, "EPSO: Evolutionary Particle Swarms", Studies in Computational Intelligence, SCI 66 (2007), pp. 139-167.

20. L. L. Garver, "Transmission net estimation using linear programming," IEEE Transactions on PAS, PAS-89 (1970), pp. 1688-1697.

21. M. C. Rocha, J. T. Saraiva, "Discrete Evolutionary Particle Swarm Optimization for Multiyear Transmission Expansion Planning", in Proceedings of the $17^{\text {th }}$ Power System Computation Conference, PSCC 2011, Stockholm, 2011.

22. IEEE Reliability Task Force, "IEEE Reliability Test System", IEEE Transactions on PAS, PAS -98 (1979), pp. 2047-2054.

23. N. Alguacil, A. Motto, A. J. Conejo, “Transmission Expansion Planning: A Mixed-Integer LP Approach”, IEEE Transactions on Power Systems, 3 (2003), pp. 1070-1077.

24. R. Romero, C. Rocha, J. Mantovani, I. Sanches, "Constructive heuristic algorithm for the DC model in network transmission expansion planning", in IEE Proceedings Generation Transmission Distribution, 152 (2005), pp. 277 - 282. 